\title{
EDITORIAL
}

\section{Allergens in foods}

\author{
Angelika Paschke $\cdot$ Franz Ulberth
}

Published online: 26 July 2009

(C) Springer-Verlag 2009

A considerable proportion of the general public experiences negative health effects triggered by certain components of their habitual diet. Susceptible persons can develop an intolerance, e.g. against lactose, owing to the lack of a digestive enzyme, some cannot tolerate certain food additives or increased levels of certain amino acids, e.g. glutamate, and others suffer from adverse reactions mediated by the immune system. The latter is characterized by the action of IgE antibodies against the offending food and is known as food allergy. The symptoms caused by allergic reactions range from rather mild manifestations (urticaria) to life-threatening events (anaphylactic shock). Therapeutic approaches to treat food allergies have been tried with variable success. Usually the recommended option is to eliminate from the diet the food(s) or food ingredients to which hypersensitivity has been found. Allergic persons need to know whether the food items they purchase contain allergenic ingredients; they have to rely on the truthfulness of the information given on the labels of prepared and packaged food items. National and supranational legislation has been put in place which requires food business operators to declare whether ingredients with a known

\footnotetext{
A. Paschke

Institute of Biochemistry and Food Chemistry,

University of Hamburg,

Grindelallee 117,

20146 Hamburg, Germany

e-mail: angelika.paschke@uni-hamburg.de

F. Ulberth $(\bowtie)$

European Commission, DG Joint Research Centre,

Institute for Reference Materials and Measurements,

Retieseweg 111,

2440 Geel, Belgium

e-mail: franz.ulberth@ec.europa.eu
}

allergenic potential were used during manufacturing. The Codex Alimentarius General Standard for the Labelling of Prepacked Food requires, for example, the mandatory labelling of the presence of eight classes of food ingredients that are known to cause hypersensitivity in susceptible consumers (cereals containing gluten, crustaceans, eggs, fish, peanuts, soybeans, milk, and tree nuts), and European Community legislation (Commission Directive 2007/68/ EC) has extended the list to include also celery, mustard, sesame seeds, lupin, and molluscs. Analytical testing systems are needed by businesses in the food industry to enable them to test whether allergens are present in their raw materials and in the finished products and whether production lines have been correctly sanitized, by food inspection authorities for market surveillance, and by academia to enable and stimulate research into food allergy and allergen detection. In this issue of Analytical and Bioanalytical Chemistry we attempted to bring together views from different angles. The majority of the reviews and original contributions focus on analytical aspects; however, biochemical, immunological, and clinical aspects are covered as well.

One of the most drastic effects an allergen contained in food may trigger is anaphylaxis; the feature article by Brockow deals with that aspect. Today, most allergic persons rely on the avoidance of the offending allergen, although other therapeutic strategies are under development (Wichers). To be able to develop an understanding for clinical as well as for diagnostic aspects of allergen research, the offending proteins need to be characterized (contributions by Hoffmann-Sommergruber and Mills, and Restani). Although most national and supranational food labelling legislation mentions eight to 14 different classes of food allergens which have to be declared, many more foods have been reported to be able to cause allergic 
reactions; Pastorello describes the outcome of double-blind placebo controlled challenge studies with maize-susceptible patients. The rest of the contributions are (bio)analytical chemistry oriented. Baumgartner gives an overview of a rapid immunoanalytical test. Immunochemistry-based tests are definitely the most widely applied tool in routine analysis as well as research laboratories for the determination of food allergens. Lateral flow devices are, in terms of simplicity of application and user-friendliness, probably the most advanced analytical tools for the rapid detection of allergens. They are widely applied in food manufacturing industries for the checking of production lines (Röder). An application of a special variant of immunochemistry, a biosensor-based system, has been applied to detect traces of hazelnut proteins in olive oil (Bremer). This is a good example where economic adulteration of food might have serious health implications. Immunochemistry is, of course, not only used for the detection of allergens in food, but also to study the interactions of allergens with the human immune system (Bernard et al.). Food processing can have a profound effect on the detectability of allergens by ELISA-based methods. Advantages but also the inherent disadvantages of the ELISA test and, in particular, the importance of validating such methods properly by including naturally incurred test materials in validation exercises are highlighted in the contribution by Taylor et al. How thermal processing changes the detectability of peanuts in a processed food matrix by using either ELISA or a PCRbased approach is reported in the article by Scaravelli et al. Consequently, alternatives to ELISA methods are needed: Kirsch et al. review mass spectrometry based methods and also touch on a very difficult aspect, which is quantification of allergens by mass spectrometry, and Weber and Godefroy used this technique for the detection of gluten markers in processed food. Whatever method a laboratory applies to detect allergens, quality assurance is a prerequisite to obtain reliable and comparable results. One critical element of external quality assurance is participation in proficiency testing rounds. The benefits of doing so are detailed by Owen.

We hope that this collection of review articles and original papers stimulates further research to better protect the well-being of consumers suffering from food allergies by improving methods for the detection of allergens in foods, by developing systems for the management of food allergies, and by a better understanding of the molecular aspects of the interaction of allergens with other food components and the effect of food processing.

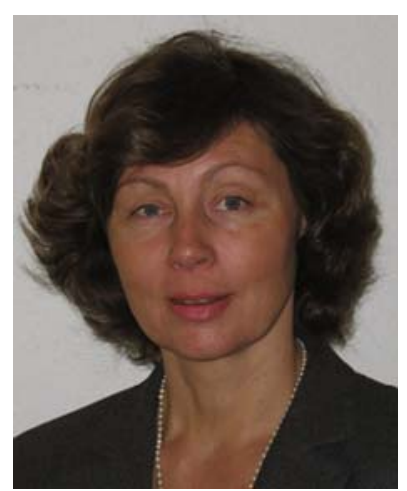

Angelika Paschke is a researcher and lecturer in food chemistry at the University of Hamburg, Germany. Her research topic is food allergy, isolation and characterization of allergens, and their alteration during technological treatment of food.

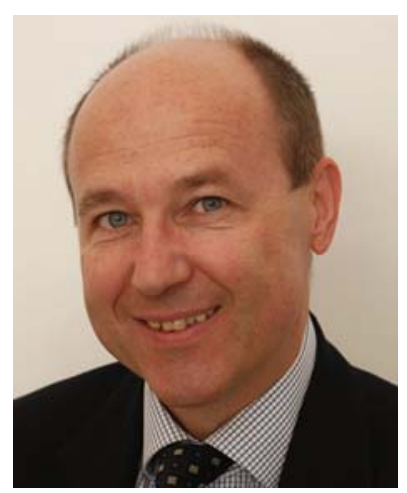

Franz Ulberth is head of the Food Safety and Quality Unit of the European Commission's Joint Research Centre, Institute for Reference Materials and Measurements, which is located in Geel, Belgium. 